\title{
Measuring amplitude and phase of light emerging from microstructures with HRIM
}

\author{
Toralf Scharf*, Myun-Sik Kim and Hans Peter Herzig \\ Ecole Polytechnique Fédérale de Lausanne (EPFL), Optics \& Photonics Technology Laboratory, \\ CH-2000 Neuchâtel, Switzerland
}

\begin{abstract}
Ultra high-resolution measurements of amplitude and phase fields emerging from fine period amplitude gratings are presented and discussed. In the axial direction periodically repeated features are found, whose origins are the Talbot effect within the Fresnel diffraction regime. The phase field recording leads to a very precise measurement of the localization of the Talbot planes and precisions below $100 \mathrm{~nm}$ are demonstrated. The concept of immersion interference microscopy is demonstrated. By accessing the back focal plane of the observation system filtering of diffraction orders provides specific Talbot images and allows to intuitively understand the role of diffraction orders for Talbot effect.
\end{abstract}

Keywords: high-resolution interference microscopy, Talbot effect, Fresnel diffraction, immersion microscopy

\section{INTRODUCTION}

Diffraction gratings and Talbot effects are intensively studied both in theoretical and experimental ways. However, the period or the size of the examined grating is relatively large because high-resolution measurement systems are required for fine gratings when periods become comparable to the operation wavelength. Scanning near-field optical microscopy (SNOM), which provides a well-known sub-diffraction-limited resolution, was applied to measure the amplitude and phase distributions emerging from a 1- $\mu \mathrm{m}$-period grating ${ }^{1}$. Although SNOM assures an increased resolution, scanning in the axial direction is somewhat difficult and filtering of diffraction orders is not feasible. In this work, we apply a highresolution interference microscopy (HRIM), whose details can be found in our previous works ${ }^{2,3}$, to investigate diffraction of light in the near-field diffraction (Fresnel diffraction) regime of the grating. High-resolution amplitude and phase fields of light emerging from 1- $\mu \mathrm{m}$ and $2-\mu \mathrm{m}$-period amplitude gratings are measured for different wavelengths. The amplitude gratings lead to highly periodic patterns in the axial direction caused by the Talbot effect. Such patterns reach periodicities of a fraction of the grating period when higher diffraction orders are contributed. We discuss the effect of wavelengths and the number of diffraction orders participating in the imaging. The Talbot effect in the Fresnel diffraction regime is probed by filtering the diffraction orders in the back focal plane of the observing objective (the Fourier plane).

Amplitude and phase fields of light emerging from periodic structures, although considered by many researchers, are still an interesting subject of research. Talbot observed the self-imaging effect of a periodic structure in $1836^{4}$. Later, Lord Rayleigh showed that its origin is the interference of the diffracted beams and found that the regular repeat distance of self-images, the so-called Talbot Length, can be expressed as

$$
Z_{T}=\frac{2 \Lambda^{2}}{\lambda}
$$

where $\Lambda$ is the grating period and $\lambda$ is the effective wavelength of the incident light ${ }^{5}$. Since smaller gratings have larger diffraction angles given by the diffraction formula as $\sin \theta=\lambda / \Lambda$, high numerical aperture (NA) and high resolution techniques are required to observe these effects for fine gratings. This is especially important for grating with periods comparable to the operation wavelength. Moreover, phase measurements need special techniques to be performed.

*toralf.scharf@epfl.ch, phone +41 32 7183286; fax +41 32 7183201; www.opt.epfl.ch

Optical Measurement Systems for Industrial Inspection VII, edited by Peter H. Lehmann,

Wolfgang Osten, Kay Gastinger, Proc. of SPIE Vol. 8082, 808210 - (c) 2011 SPIE

CCC code: $0277-786 X / 11 / \$ 18 \cdot$ doi: $10.1117 / 12.895011$

Proc. of SPIE Vol. 8082 808210-1 


\section{EXPERIMENT}

The HRIM is basically a microscope with objectives that have the highest numerical apertures combined with a MachZehnder interferometer. It allows to apply all conventional microscopy imaging techniques for transmission, such as immersion or polarization. Immersion can be used to increase spatial resolution and polarization is normally applied as a contrast enhancement technique. We show below the influence of different operation wavelengths and we present results obtained both in air and in immersion.

High-resolution interference microscopy has been already proven as a powerful tool for characterizations of micro-size optical elements, such as microlenses and gratings ${ }^{2,3}$. HRIM is working in transmission by employing a Mach-Zehnder type interferometer as shown in Fig. 1. Three single mode polarized laser diodes (CrystaLaser, 642nm: DL640-050-3, 532nm: IR-GCL-025-S, 405nm: BCL-040-405-S) with different powers and wavelengths are used to investigate the wavelength dependence in visible spectrum. A polarizing beam splitter (PBS) divides intensities to be sent in a reference and an object arm with adjustable energy ratio. Half wave plates (HWP) and Glan-Taylor (G-T) polarizers are applied to adjust the intensities and to optimize the contrast of the interference fringes. In the object arm, an expanded collimated beam (a plane wave) illuminates the system under investigation, which is mounted on a precision piezo stage with a $\mathrm{z}$ scan range of $500 \mu \mathrm{m}$ and a nominal accuracy of $1 \mathrm{~nm}$ (MAD LAB CITY, NANO Z500). This z-axis piezo stage allows us to precisely find the plane of interest at the highest resolution. In the reference arm, a piezo-electrically driven mirror is mounted to change the optical path lengths. The phase distribution of the wave field is obtained by measuring the interference fringes at different mirror positions and employing a classical 5-frame algorithm, which is termed SchwiderHariharan method ${ }^{6,7}$. In this scheme, 5 frames of the intensity pattern are recorded, each frame being shifted in phase by adding an additional phase of $\lambda / 4$ by moving the mirror in the reference arm. The phase fields are evaluated numerically and phase unwrapping procedures might be applied if it is necessary. This interferometric function allows measuring the phase distributions of the incident illumination beam and the emerging light fields after microstructures, which are gratings in our case.

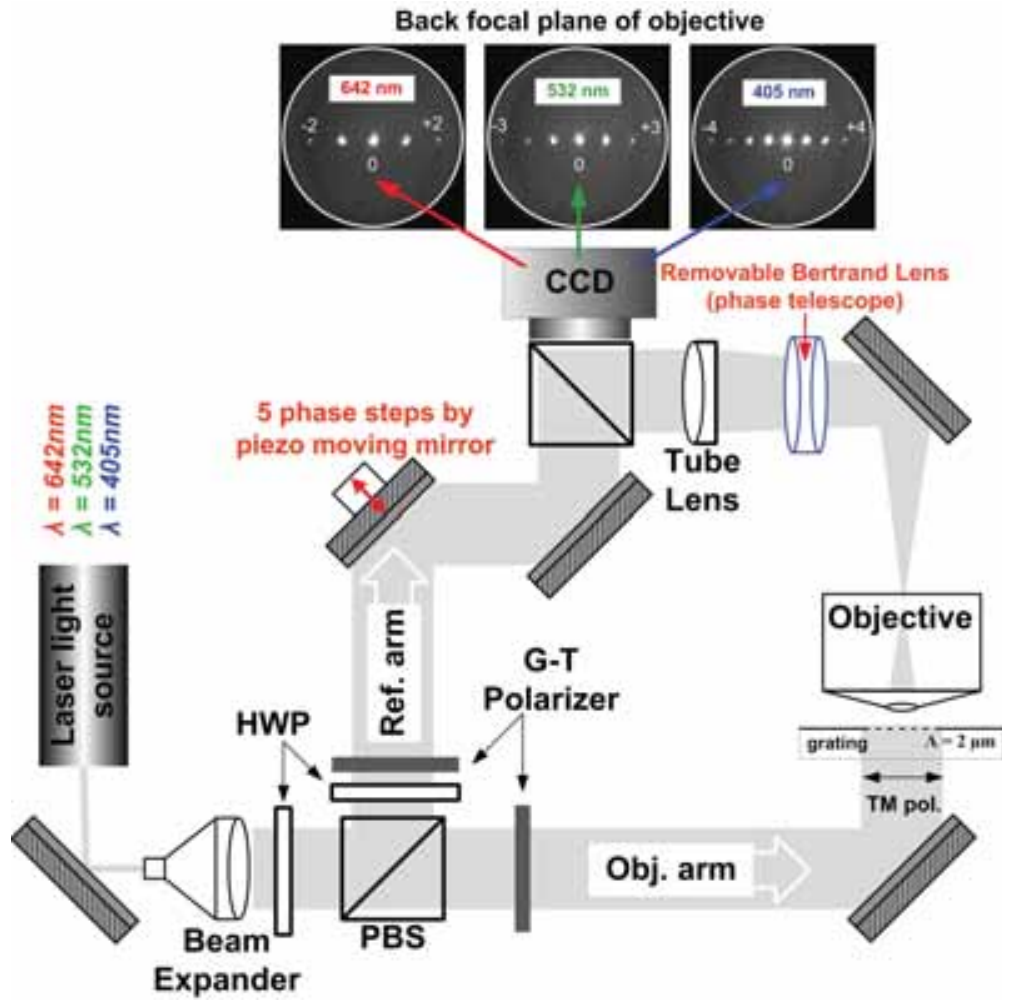

Figure 1. Schematic of the experimental setup with Bertrand lens setup. In the reference arm the piezo driven mirror modulates the optical path with 5 steps of $\lambda / 4$. A Bertrand lens can be placed in the object arm to visualize the participation diffraction orders. This is particular useful for investigations of gratings. Insets show the CCD images of the back focal plane at three different wavelengths incidence to the $2-\mu \mathrm{m}$ grating. 
In general, a high numerical aperture (NA) of the observation objective ensures high-resolution of the amplitude and phase measurements. Moreover, high magnification provides more pixels on an image sensor for small fields. We use highly corrected large NA objectives, e.g, a 100X / NA 0.9 dry objective (Leica Microsystems, HC PL FLUOTAR). The resolution of an optical microscope is usually defined as the minimum resolvable distance between two points based on the Rayleigh criterion ${ }^{8}$. The lateral resolution for this objective is $\Delta \mathrm{x}=435 \mathrm{~nm}$, which is calculated with the formula $\Delta \mathrm{x}$ $=0.61 \lambda / \mathrm{NA}$ with a wavelength of $642 \mathrm{~nm}$. Along the optical axis, the Rayleigh criterion can be applied with the simplified formula derived as $\Delta \mathrm{z}=\lambda \cdot \mathrm{n} /\left(\mathrm{NA}^{2}\right)$ with $\mathrm{n}$ the refractive index of the object space ${ }^{9}$. The calculated axial resolution at $642 \mathrm{~nm}$ wavelength in air is $\Delta z=793 \mathrm{~nm}$. At 100X magnification, a pixel on a charge-coupled device (CCD) sensor (Sicon Corporation, CFW1312M camera with SONY ICX205AK image sensor of 1360 x 1024 pixels) corresponds to $46.5 \mathrm{~nm}$ in the object space. This leads to the maximum field of view of the CCD camera of $64 \times 48 \mu \mathrm{m}^{2}$, which is large enough to image the sufficient number of periods of the grating.

In Fig.1 the optional Bertrand lens allows to visualize the back focal plane and show the participating diffraction orders for the image space. The insets of Fig. 1 show diffraction orders of a $2-\mu \mathrm{m}$ grating for three different wavelengths observed with a $100 \mathrm{X} / 0.9$ dry objective. For a $2-\mu \mathrm{m}$-period grating at normal incidence one finds the angle for the first diffraction order as $19^{\circ}$ for $642 \mathrm{~nm}, 15^{\circ}$ for $532 \mathrm{~nm}$, and $12^{\circ}$ for $405 \mathrm{~nm}$. The observing objective has a numerical aperture of NA $=0.9$ corresponding to a maximum acceptance angle of $64^{\circ}$. Hence, diffraction orders from 0 to $+/-3$ can be collected by such an objective at $642 \mathrm{~nm}$ illumination. For $532 \mathrm{~nm}$ and $405 \mathrm{~nm}$, higher diffraction orders from 0 to $+/-$ 4 and from 0 to $+/-5$ contribute, respectively. In practice, intensity falls off for large angles and less diffraction orders might be collected because certain experimental errors limit the NA of the objective to be smaller than 0.9 . The number of diffraction orders gets fewer as the wavelength increases, as shown in Fig. 1. Therefore, better contrast and higher resolution are obtained for the intensity measurement at a shorter wavelength, in our case $405 \mathrm{~nm}$, thanks to the contributed higher orders.

\section{LIGHT FIELDS EMERGING FROM AMPLITUDE GRATINGS FOR DIFFERENT WAVELENGTHS}

Three different wavelengths, red at $642 \mathrm{~nm}$, green at $532 \mathrm{~nm}$ and blue at $405 \mathrm{~nm}$, are applied to investigate the wavelength dependence of the diffraction angle and the Talbot length. We considered as illumination a plane wave $(\lambda=$ $642 \mathrm{~nm}$ ) propagating along the positive $z$-direction and being polarized in the $x$-direction, which is the TM case of the grating. Examples of the periodic patterns in the axial direction are given in Fig. 2 as presented in the X-Z slices of measured 3D intensity distributions from the $2-\mu \mathrm{m}$-period amplitude grating for three wavelengths. One observes selfimages at $Z_{\mathrm{T}}$ and shifted patterns by half period at the half of the Talbot length $\left(\mathrm{Z}_{\mathrm{T}} / 2\right)$ as expected from theory.

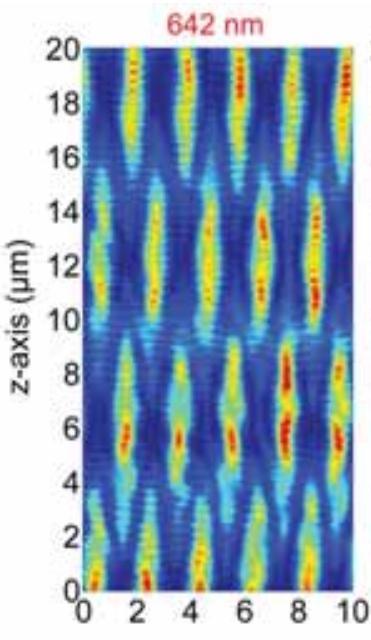

(a)

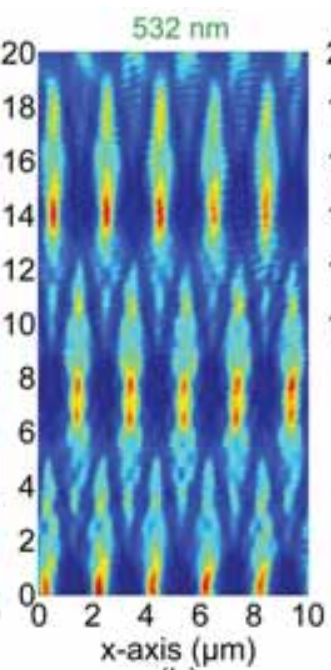

(b)

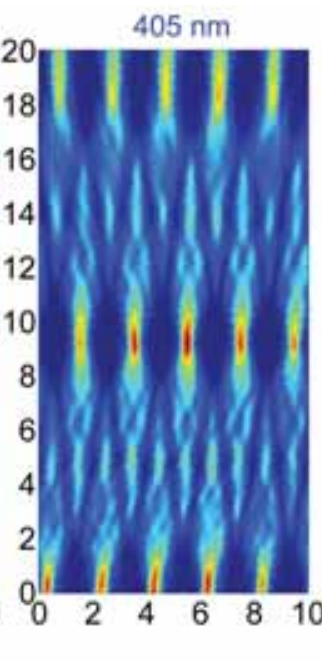

(c)

Figure 2. The x-z slices of measured 3D intensity distributions (10 x $20 \mu \mathrm{m}^{2}$ ): (a) $642 \mathrm{~nm}$, (b) $532 \mathrm{~nm}$, and (c) $405 \mathrm{~nm}$. Determining the Talbot lengths in these images is subjected to experimental uncertainty because of the diffraction-limited observation when only intensity fields are analyzed. 
The visibility of the smaller fractions of the Talbot images differs depending on the illuminating wavelength because the number of collected diffraction orders varies for different wavelengths. To obtain more information and precisely localize the Talbot planes the phase of the emerging fields are investigated by applying the aforementioned interferometric technique. Figures 3(a) - 3(c) show the $x-z$ slices of the measured 3D phase data for the three wavelengths. At the multiple of the half of the Talbot length $\left(\mathrm{Z}_{\mathrm{T}} / 2\right)$, one finds planes with particular phase structures, i.e., phase singularities. Because of the extremely low intensity near planes where singularities are located, measurement noise is amplified considerable. Compared to the intensity measurements, the phase distributions reveal much more details. For example, the precision of the position measurement of singularities is given by the pixel resolution of the instrument. More precise evaluations of the Talbot length can be carried out by measuring the distance between planes where phase singularities occur. By analyzing Fig. 3 and measure the Talbot distance for different wavelengths one finds for $642 \mathrm{~nm}, 532 \mathrm{~nm}$ and $405 \mathrm{~nm}, Z_{\mathrm{T}}=12.2 \mu \mathrm{m}, \mathrm{Z}_{\mathrm{T}}=14.4 \mu \mathrm{m}$, and $\mathrm{Z}_{\mathrm{T}}=19.9 \mu \mathrm{m}$, respectively. The Talbot lengths can be calculated using Eq. (1) to be $12.5 \mu \mathrm{m}$ for $642 \mathrm{~nm}, 15 \mu \mathrm{m}$ for $532 \mathrm{~nm}$, and $19.8 \mu \mathrm{m}$ for $405 \mathrm{~nm}$. Experimental value and show very good agreement with the analytical values from Eq. (1). Measurement errors of the HRIM for this measurement are estimated to be $0.1 \mu \mathrm{m}$, given by the size of two steps in the $z$-direction of the measured voxel.

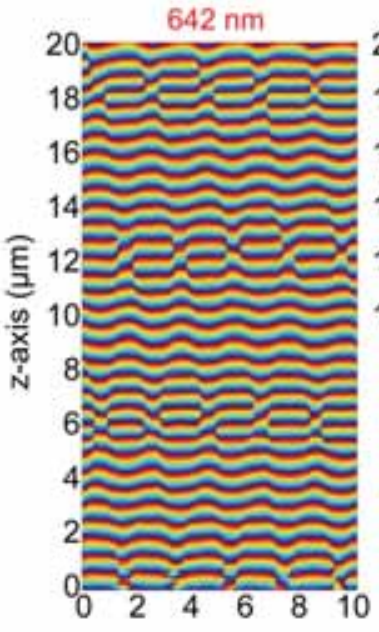

(a)

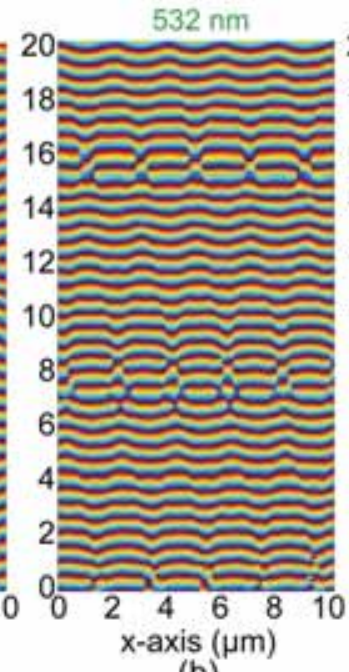

(b)

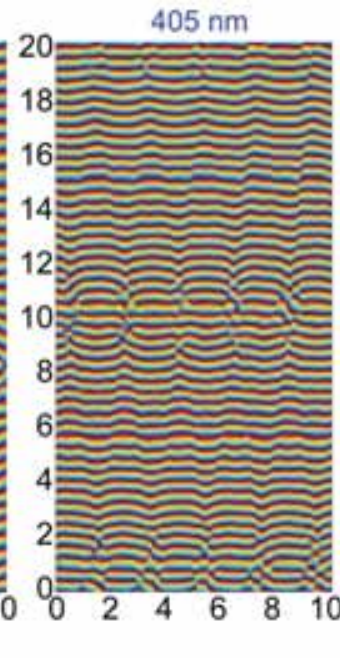

(c)

Figure 3. The $x-z$ slices of the measured 3D phase distributions (10 x20 $\mu \mathrm{m}^{2}$ ): (a) $642 \mathrm{~nm}$, (b) $532 \mathrm{~nm}$, and (c) $405 \mathrm{~nm}$. At the multiple of the half Talbot length $\left(\mathrm{Z}_{\mathrm{T}} / 2\right)$, the self-images of the plane wave surrounded by the phase singularities are observed. Distance between such planes serves as a precise measure of the Talbot length.

\section{IMMERSION INTERFERENCE MICROSCOPY}

Resolution in a diffraction-limited imaging system can be increased by using immersion techniques. Such technique cannot be applied under all circumstances because the immersion might destroy optical phase shift created by dielectric structures. For measurements of light fields generated by amplitude gratings, however, this is an ideal mean to increase resolution. For immersion, the space between the observing objective and the object is filled with an immersion liquid of high refractive index. Special objectives corrected for these techniques have to be applied. We used a 100X / NA0.7-1.4 oil objective from Leica Microsystems (HXC PL APO) to collect light in different numerical apertures which can be adjusted between NA $=0.7$ and 1.4 continuously. Immersion medium is a standard immersion oil with the refractive index of $\mathrm{n}_{\mathrm{D}}=1.515$ (Leica Microsystems, Type $\mathrm{N}$ immersion liquid). The immersion leads to a larger effective numerical aperture and therefore more diffraction orders are collected as illustrated in Fig. 4. Figure 4 gives a comparison between the measurements in air and oil immersion and the number of diffraction orders can be counted when observing the back focal plane with the Bertrand lens setup.

Analysis of the intensity measurements in Fig. 4 reveals that the immersion effect allows to identify the sub-Talbot image planes because of its higher resolution. These planes show structured light fields with the double of the periodicity of the original amplitude grating, here $2 \mu \mathrm{m}$. In the case of immersion on the right of Fig. 4, sub-Talbot planes are found at about $z=5 \mu \mathrm{m}$ and $z=15 \mu \mathrm{m}$ distance from the grating surface $(z=0)$. 


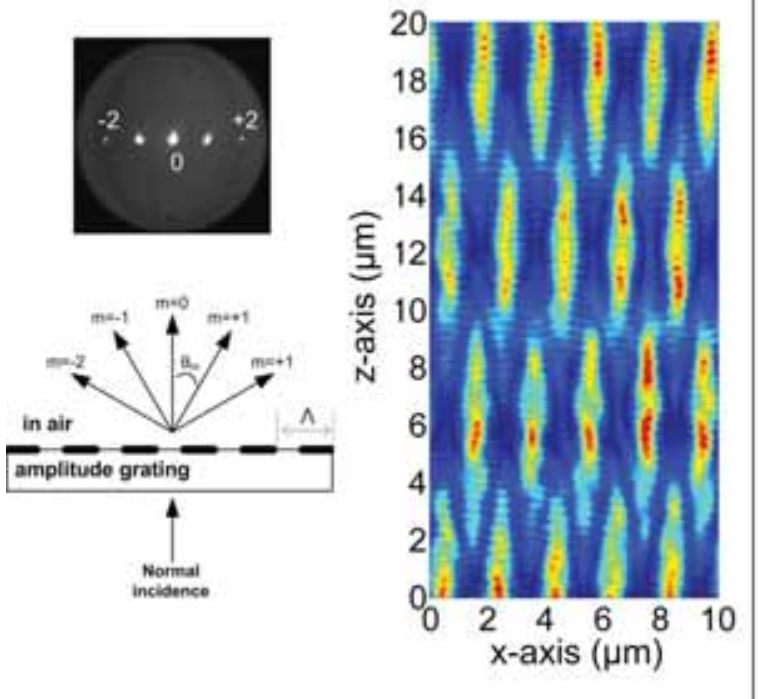

(a)

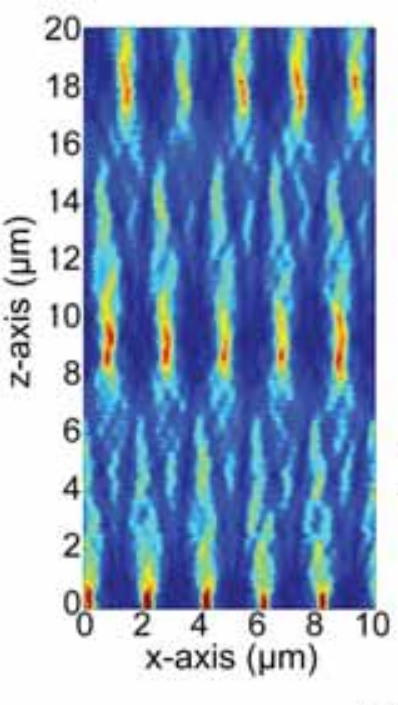

(b)
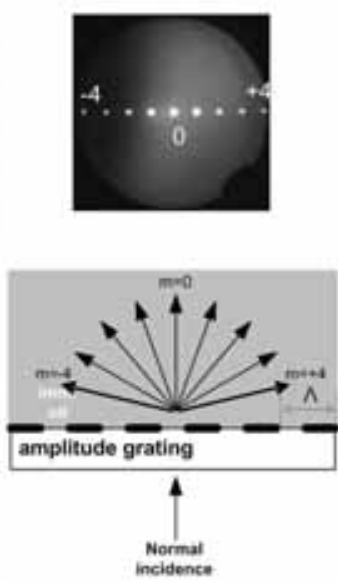

Figure 4. Measurement example for the immersion effect on an amplitude measurement. (a) The case without immersion when the medium between objective and sample is air $(n=1)$. (b) Oil immersion situation with an immersion oil that has a refractive index of $n_{D}=1.515$. The insets show the diffraction orders of the $2-\mu \mathrm{m}$ grating when the surrounding medium is air or immersion oil. The immersion leads to the increased number of diffraction orders and a higher resolution in the amplitude image.

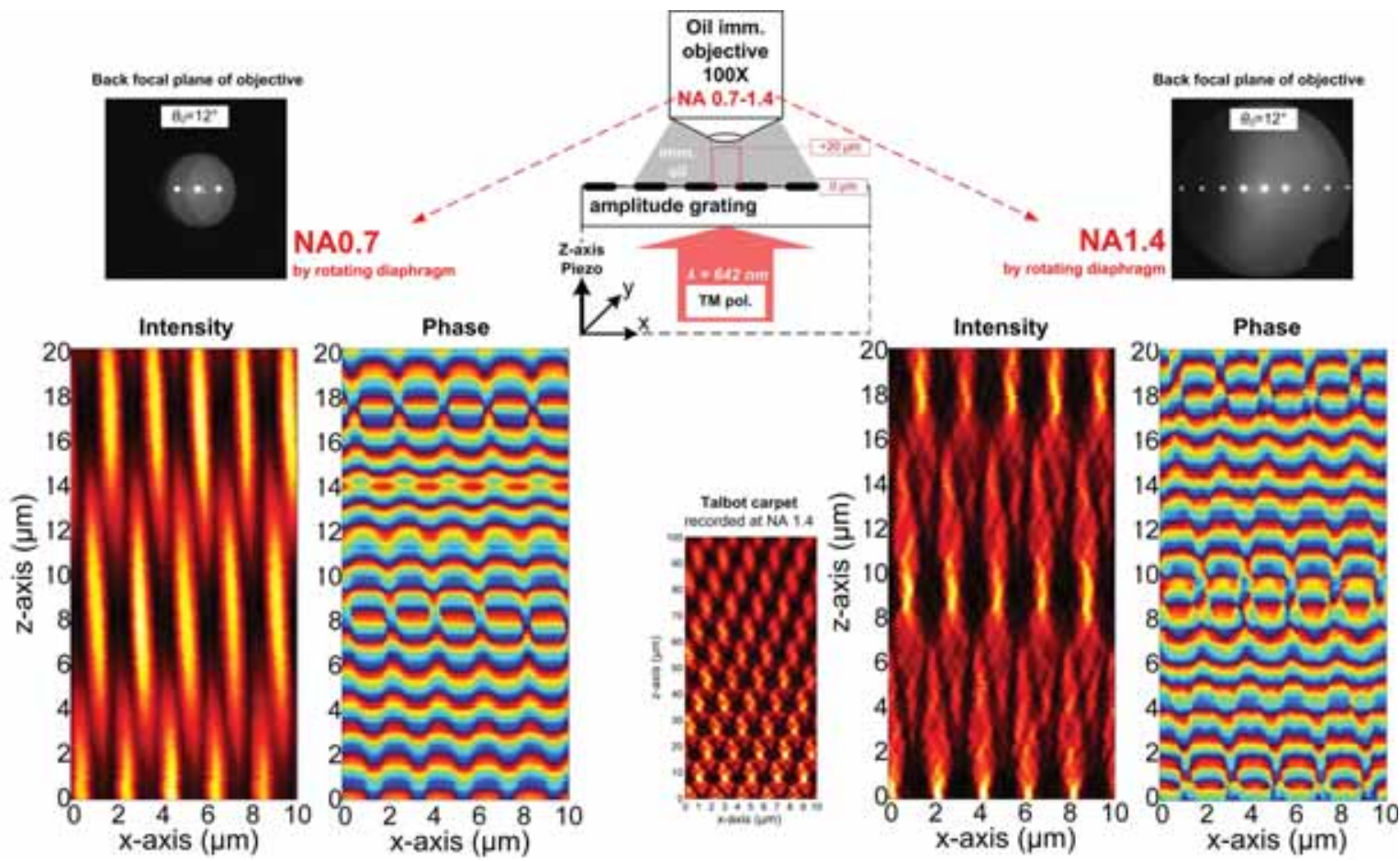

Figure 5. Influence of the numerical aperture on amplitude and phase measurements of Talbot carpets. All measurements are performed in immersion. On the left the measurement with low NA of 0.7 and on the right high NA case with NA $=1.4$. CCD images of the back focal plane for each case are shown in the insets. 
We can adjust the numerical aperture with our immersion objective without changing other experimental conditions in the setup. Technically the numerical aperture is set by opening and closing a diaphragm, which is integral part of the objective. It is instructive to see how resolution in intensity and phase field increasing when switching from $\mathrm{NA}=0.7$ to $\mathrm{NA}=1.4$. Figure 5 provides an example for such arrangements with the $2-\mu \mathrm{m}$ amplitude grating. In immersion $(\mathrm{n}=1.51$ at $\lambda=642 \mathrm{~nm})$ the effective wavelength inside medium becomes $\lambda_{\text {medium }}(=\lambda / \mathrm{n})=425 \mathrm{~nm}$, which changes the period of the wave field. To evaluated the Talbot distance we applying this effective wavelength in Eq. (1) with an period of the grating of $\Lambda=2 \mu \mathrm{m}$ and find the Talbot distance $Z_{T}=18.8 \mu \mathrm{m}$. Having a close look on Fig. 5 we see that the intensity images suffer from a reduced resolution and a smoothing of the intensity images is observed as the consequence of the resolution decrease. Although the phase fields are also smoothed, they still keep the information about the planes of singularities encoded at very high precision. When we evaluated the Talbot distances in both cases, we could not find substantial difference that falls out of the measurement precision; assumed to be the double of the $z$-axis pixel size of the 3D measurement. Immersion leads to better resolution because of the contribution of higher spatial frequencies. In the phase field measurements the higher spatial frequencies allow us to see larger curvatures (smaller radius of curvature) of wavefronts surrounding the isolated plane-wave island in the middle of two singularities. This is clearly visible by analysing special areas in Fig. 5 such as zones of phase distributions around singularities.

\section{TALBOT EFFECT IN THE FRESNEL DIFFRACTION REGIME}

We studied diffraction of light from an even finer grating than $2-\mu \mathrm{m}$ period with the aim to analyze Talbot effects when the periodicity becomes very close to the operation wavelength. In our experiments, a plane wave of $642 \mathrm{~nm}$ illuminates a $1-\mu \mathrm{m}$-period amplitude grating. The space behind the grating is immersed in oil in order to provide the highest effective numerical aperture and the best resolution for this experimental arrangement. Close to the grating, in the socalled Fresnel diffraction regime, interference of diffracted beams forms periodic features in the axial direction as shown in Figs. 6(a) and 6(b). These patterns are directly associated to the Talbot effect. Different situations can be simulated in this measurement. For an easier interpretation, the participating diffraction orders in the back focal plane are visualized as CCD images in the top row of Fig. 6.

The immersion-HRIM provides a sufficient axial resolution to verify the Talbot length of $4.7 \mu \mathrm{m}$ approximated by Eq. (1). Figures 6(a) and 6(b) show the influence of varying the numerical aperture for the 1- $\mu \mathrm{m}$-period grating. Again one finds the enhanced resolution in intensity images and larger curvatures (smaller radius of curvature) in phase distributions for images containing higher orders such as Fig. 6 (b). Additional consequences linked to the limitation of the measurement system are observed. When the focal plane of the objective moved away from the grating surface during the 3D scanning, light diffracted under high angles propagates out of the angle of acceptance cone of the objective. Under such circumstances the Talbot carpet will show variations of its appearance when observed along the $z$ direction. Figure 6 (b) makes this effect clear when the intensity distributions close to the grating (at position $\mathrm{z}=0 \mu \mathrm{m}$ ) and far from the grating (at position $\mathrm{z}=20 \mu \mathrm{m}$ ) are compared. It seems that the resolution is decreased for the latter position by an effect that is caused by the missing contributions from higher diffraction orders that have already propagated out of the acceptance angle. Since low diffraction orders like the first orders have a smaller diffraction angle, within the relative short measurement distance in the z-axis this effect is nearly invisible as shown in Fig. 6(a).

In the back focal plane of the objective, diffraction orders can be selectively removed by a spatial filtering technique. In this way patterns appearing in the image space can be influenced. Such filtering will create artificial interference situations and examples are shown in Figs. 6(c) and 6(d). Without the $0^{\text {th }}$ order self-images are not formed. Having only the $-1^{\text {st }}$ and $+1^{\text {st }}$ orders interference patterns with stripes happen. Note that this is not the real field behind the grating but the artificial images as the filtering of diffraction orders is performed after the propagation through the grating jut before the image plane. The phase field shows typical interference patters with two plane waves $\left(-1^{\text {st }}\right.$ order wave and $+1^{\text {st }}$ order wave) travelling at a certain angle, the double of the diffraction angle. Observing far away from the grating, contrast decreases due to the loss of the higher diffraction orders as discussed before. In Fig. 6 (d) we have the same findings for the case of the four-wave interference, hence the participations are $-2,-1,+1$ and +2 diffraction orders. Still the zeroth order is cut off. The recorded intensity and phase patterns are more complex but reveal the same features as found in the 2 -wave interference case. It becomes clear that a minimum of three diffraction orders including the $0^{\text {th }}$ order are necessary to find the Talbot effect as shown in Fig. 6 (a). Additional higher diffraction orders are associated with the birth of the so-called sub-Talbot features. In the Fresnel diffraction regime waves retain properties of the incident beam in our case plane waves. That is the cause why the particular 2-plane-wave interference given in Fig. 6(c) leads to simple intensity lines in the image space. 


\section{Back focal plane}
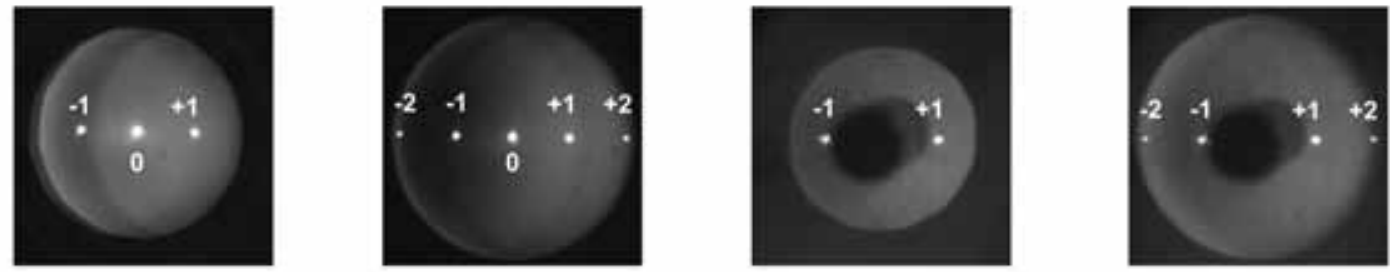

Intensity
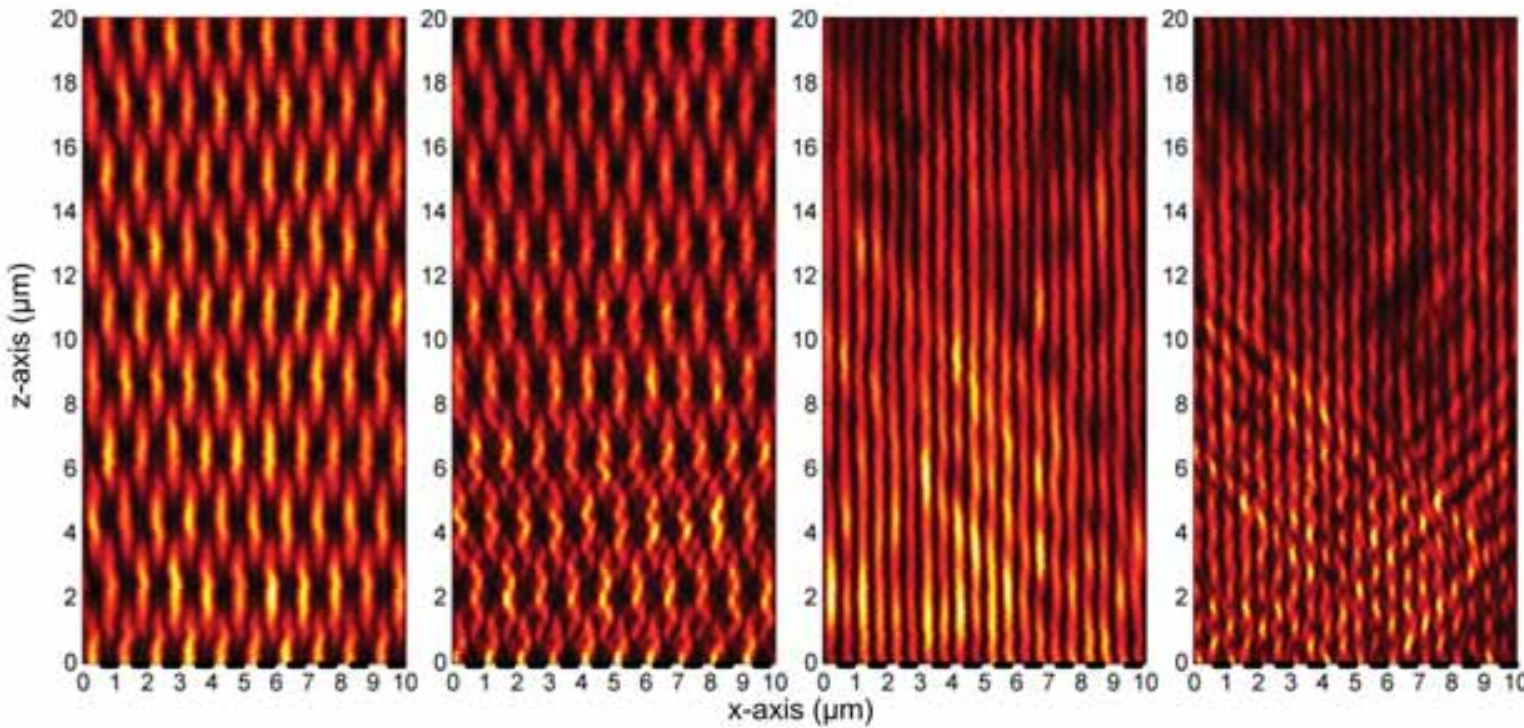

Phase

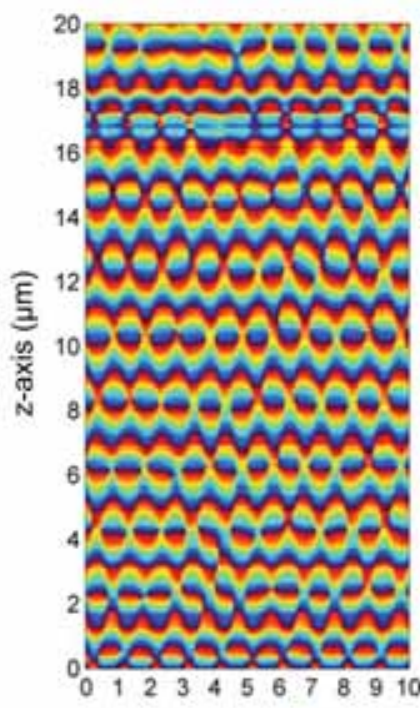

(a)

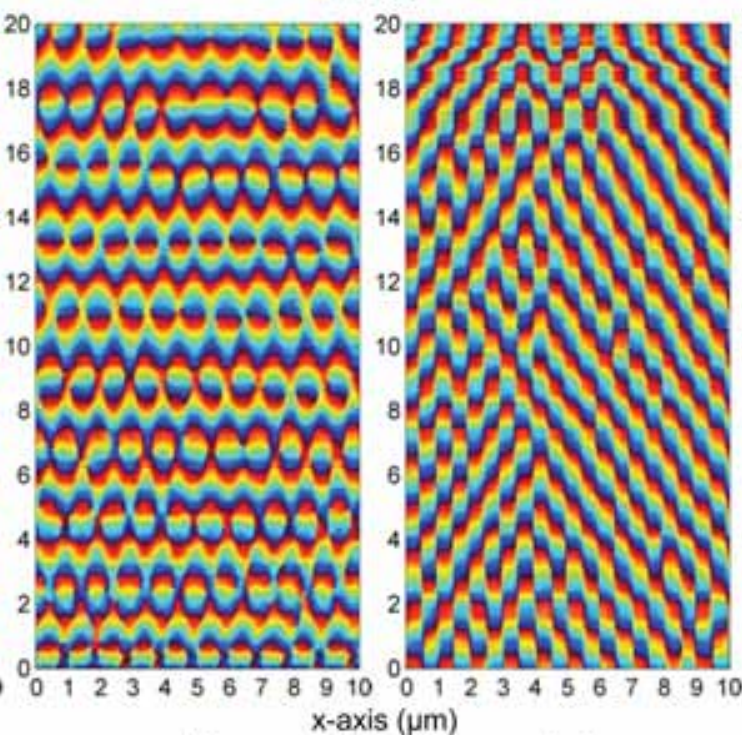

(b)

(c)

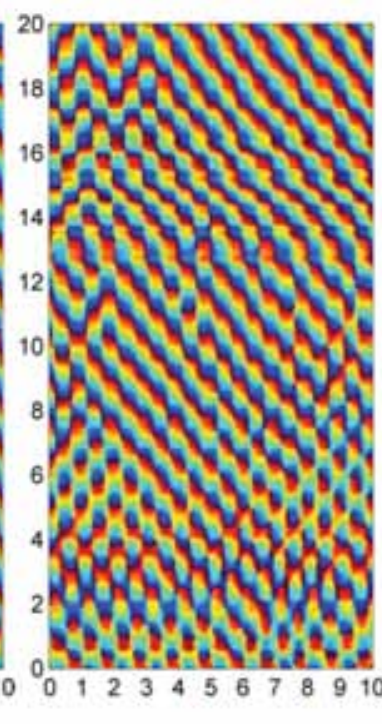

(d)

Figure 6. Measured light fields for different diffraction orders merging form a 1- $\mu \mathrm{m}$ grating at $642 \mathrm{~nm}$ wavelength. The upper row: CCD images of the back focal plane of the objective to illustrate the participating diffraction orders. The dark disc in (c) and (d) is used to block the 0th order. The middle row gives intensity distributions in the $x-z$ plane and the lower row presents the corresponding phase distributions: (a) $0, \pm 1$ orders, (b) $0, \pm 1$, \pm 2 orders, (c) \pm 1 orders, and (d) $\pm 1, \pm 2$ orders. 


\section{CONCLUSIONS}

The HRIM allows ultra high-resolution measurements of intensity and phase distributions. Especially, the phase measurement provides more complete descriptions of light propagation emerging from microstructures. Our experimental investigations reveal a multitude of details like phase singularities that can be used for characterization and measure of microstructures when direct imaging is not anymore useful. We have shown that even under low numerical aperture conditions singularities can be localized at high precision with the help of the phase fields. Even in the close vicinity of fine gratings, e.g., 1- $\mu \mathrm{m}$ period, where the Fresnel diffraction dominates, the Talbot effect is present due to the wave nature of light. We have given illustrative examples for different wavelengths and showed that immersion interference microscopy opens a pathway to high-resolution measurements and positioning techniques when applied to small microstructures like gratings.

\section{ACKNOWLEDGEMENT}

The research leading to these results has received funding from the European Community's Seventh Framework Programme FP7-ICT-2007-2 under grant agreement No. 224226.

\section{REFERENCES}

[1] Nesci A., Dändliker R., Salt M., and Herzig H. P." Measuring amplitude and phase distribution of fields generated by gratings with sub-wavelength resolution", Opt. Comm. 205, 229, 2002

[2] Rockstuhl, C., Märki, I., Scharf, T., Salt, M., Herzig, H. P. and Dändiker, R., "High Resolution Interference Microscopy: A Tool for Probing Optical Waves in the Far-Field on a Nanometric Length Scale," Current Nanoscience 2, 337-350 (2006).

[3] Kim, M.-S., Scharf, T. and Herzig, H. P., "Small size microlenes characterization by Multiwavelength High Resolution Interference Microscopy," Opt. Express 18, 14319-14329 (2010).

[4] Talbot F., "Facts relating to optical science. No. IV", Philos. Mag. 9, 401, 1836

[5] Rayleigh L., "On copying diffraction gratings and some phenomena connected therewith", Philos. Mag. 11, 196, 1881

[6] Schwider, J., Burow, R., Elssner, K.-E., Grzanna, J., Spolaczyk, R. and Merkel, K., "Digital wave-front measuring interferometry: some systematic error sources," Appl. Opt. 22, 3421-3432 (1983).

[7] Hariharan p., B. F. Oreb, and T. Eiju, "Digital phase-shifting interferometry: a simple error-compensating phase calculation algorithm," Appl. Opt. 26, 2504-2506 (1987)

[8] Born, M. and Wolf, E., [Principles of Optics, 7th (expanded) edition], Cambridge University Press, Cambridge, Chap 7 and 9 (1999).

[9] Gross, H., [Handbook of Optical Systems, vol. 3] Wiley-VCH, Weinheim, p. 126 (2007). 